\title{
Latest Results on Cosmic Ray Spectrum and Composition from Three Years of IceTop and IceCube
}

\author{
The IceCube Collaboration ${ }^{1}$, T. Feusels ${ }^{2}$ \\ ${ }^{1}$ http://icecube.wisc.edu/collaboration/authors/icrc15_icecube \\ ${ }^{2}$ University of British Columbia, Department of Physics and Astronomy \\ E-mail: krawlins@uaa.alaska.edu
}

\begin{abstract}
With the IceTop detector at the South Pole, a spectrum of cosmic ray air shower size $S_{125}$ can be unfolded into an energy spectrum of primary cosmic rays. When the IceTop data is analyzed in coincidence with high-energy muon energy loss information from the deep IceCube detector, both the spectrum and mass composition of primary cosmic rays can be extracted using a neural network. Both of these analyses have been applied to three years of IceTop and IceCube data: from mid-2010 to mid-2013, using the 73-station/79-string and 81-station/86-string detector configurations. Both analyses are now sensitive to energies from $2.5 \times 10^{15} \mathrm{eV}$ to $1.2 \times 10^{18} \mathrm{eV}$. The performance and relative advantages of the two analyses will be discussed, and both all-particle spectra as well as individual spectra for elemental groups will be presented.
\end{abstract}

Corresponding authors: K. Rawlins ${ }^{\dagger *}$

$\dagger$ University of Alaska Anchorage, Department of Physics and Astronomy

The 34th International Cosmic Ray Conference,

30 July- 6 August, 2015

The Hague, The Netherlands

\footnotetext{
* Speaker.
} 


\section{Introduction}

The IceCube Observatory consists of both a surface component and a deeply-buried component. The "in-ice" neutrino telescope (described in detail in [1]) consists of 5160 Digital Optical Modules (DOMs) arranged on 86 strings at 1450-2450m depths in $1 \mathrm{~km}^{3}$ volume of Antarctic ice. The surface array "IceTop" (described in detail in [2]) consists of 162 ice Cherenkov tanks containing two DOMs arranged as 81 stations, covering an area of $1 \mathrm{~km}^{2}$. This work will focus on two analyses: an IceTop-alone analysis to measure the all-particle cosmic ray energy spectrum, and an IceTop-IceCube coincidence analysis which can measure both the spectrum and composition.

In [3], one year of data from the 73-station IceTop detector (IT73) alone was analyzed using shower size as a proxy for primary energy, and measured a spectrum from a few $\mathrm{PeV}$ to $1 \mathrm{EeV}$. With coincidence events, surface observables from IceTop can be combined with muon bundle energy loss observables from the in-ice detector. In [4], one month of 40-station/40-string coincidence data was used to measure a spectrum and average $\log$ mass $\langle\log A\rangle$ from 1 to $30 \mathrm{PeV}$; an improved analysis was performed in [5, 6] using one year of 73-station/79-string (IT73-IC79) data, achieving better resolution and reaching to $1 \mathrm{EeV}$. In this work, both analyses are extended to 3 years of data.

\section{Data and Reconstruction}

\subsection{The 3-year Dataset}

The analyses described here use the same dataset: from June 1, 2010, until May 2, 2013, with a total livetime of 977.6 days. The first year of this data was taken in IceCube's IT73-IC79 configuration. For the second and third years, IceCube was running in its complete configuration of 81 stations and 86 strings. In order to analyze the three years together and compare to Monte Carlo simulations of the IT73-IC79 configuration, the IT81-IC86 data was "retriggered" to the slightly smaller IT73-IC79 configuration.

\subsection{Reconstructions}

Data from IceTop tanks are put through a reconstruction procedure which has been described in detail in [2]. For each event, the best-fit shower core position $\left(x_{c}, y_{c}, z_{c}\right)$ and direction $(\theta, \phi)$ is found, as well as two parameters describing the shape of the lateral distribution function (LDF) of deposited charge $\left(S_{125}, \beta\right) . S_{125}$ is the signal strength measured in vertical equivalent muons (VEM) at a reference distance of 125 meters, which is the average distance between stations. This shower size parameter is a proxy for primary energy with only minor composition sensitivity, as shown in Figure 1(left). $\beta$ is related to the slope of the LDF.

Signals in the surface detectors are attenuated by snow, which accumulates unevenly over the array over time. The reconstruction accounts for snow attenuation through a simple exponential reduction applied to the expected charge, which depends only on the slant depth of snow overburden for the tank, and an "effective attenuation length" $\lambda$. As the snow load increases from year to year, the total signals in IceTop become on average more muonic, and $\lambda$ (which describes the effective attenuation of the total signal) is expected to change. So, each of the three years was optimized separately to find the $\lambda$ which best creates agreement in the $S_{125}$ spectrum across different regions 
in the array with different snow coverages. These best values of $\lambda$ are: 2.1 meters for 2010/11, 2.25 meters for 2011/12, and 2.25 meters for 2012/13.

In the coincidence analysis, the energy loss pattern of the high-energy muon bundles in the IceCube strings is reconstructed using a technique discussed in detail in [7]. For each event, a detector response matrix is obtained from tables derived from simulations and parametrized using spline-fits. Inverting the detector response matrix allows the energy loss profile as a function of slant depth to be determined from the pattern of hits.

The energy loss profile is then fit, to extract a) the average energy loss behavior and b) the size and quantity of deviations from that average behavior due to stochastic losses (the "stochastics"). The energy loss $d E_{\mu} / d X$ at a fixed slant depth of $X=1500 \mathrm{~m}$, which corresponds roughly to the top of the IceCube detector, is a highly composition sensitive observable, as shown in Figure 1(right).
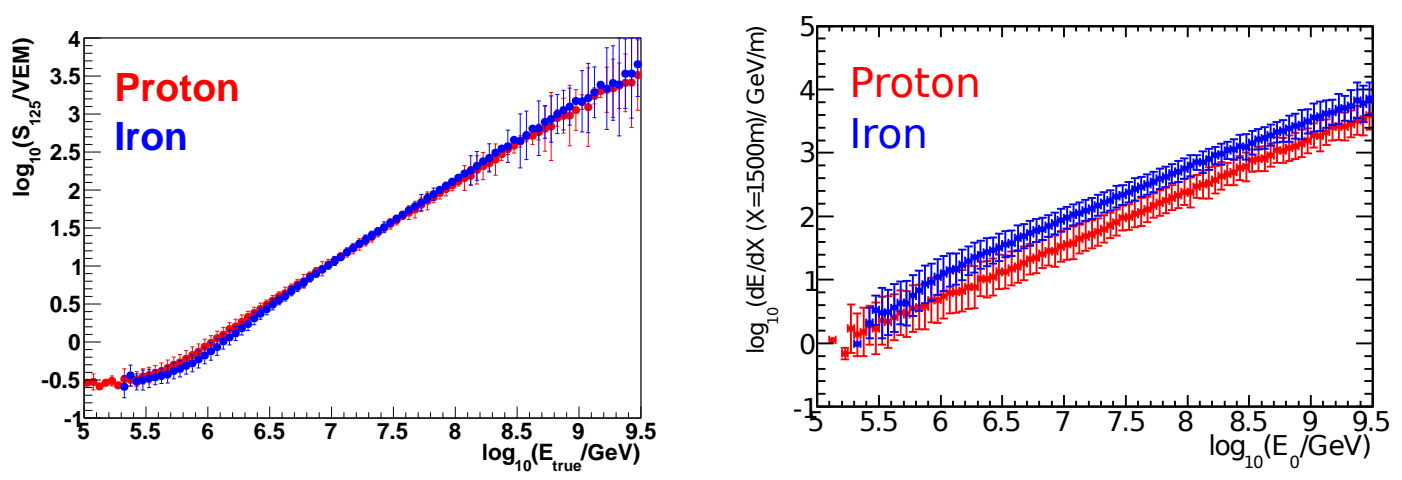

Figure 1: Left: $S_{125}$ as a function of primary energy, which has minimal sensitivity to composition. Right: $d E_{\mu} / d X$ at $1500 \mathrm{~m}$ as a function of primary energy, which is highly composition-sensitive.

The number of high-energy stochastics is also composition-sensitive. Iron bundles have more stochastics because the bundles contain more muons, despite that the energy losses from proton bundles can be more extreme. Two methods of selecting a number of high-energy stochastics from an energy loss profile are used in this work: a standard selection and a strong selection requiring higher stochastic energy loss. The standard stochastics count is composition sensitive at low energies. Above $100 \mathrm{PeV}$ where the standard selection loses sensitivity, the strong selection becomes sensitive.

Changes in atmospheric temperature from summer to winter produce a measured variation in $\log _{10}\left(d E_{\mu} / d X\right)$. The magnitude of the variation is $10-15 \%$ of the difference between protons and iron in Figure 1(right). Simulations represent one atmosphere (from July, 1997), and all other months of data are corrected with respect to July, using a measured relationship between the temperature profile of the entire atmosphere, the muon production depth profile, and the measured variation of $\log _{10}\left(d E_{\mu} / d X\right)$. Applying this correction reduces the variation to $\pm 3 \%$ of the difference between protons and iron. For more details, see [15].

\subsection{Quality Cuts}

The IceTop quality cuts for the IceTop-alone and Coincidence analyses were unified, based 
on the cuts described in [3] and [5,6], requiring 5 hit stations, a converged reconstruction, and a slope parameter $\beta$ between 1.4 and 9.5. To remove uncontained events, the "loudest" station must not be on the edge, and must have a charge of at least 6 VEM. Because the coincidence analysis is very sensitive to contamination from uncontained events and other rare outliers, some additional quality cuts are applied on the surface events. Additionally for coincidences, the track position and direction (determined by IceTop), is required to pass within 0.96 of the volume of the in-ice detector perimeter, and several additional cuts ensure the quality of the energy loss fit. In both analyses, the reconstructed core position is required to be contained within IceTop.

After reconstruction and cuts, the event

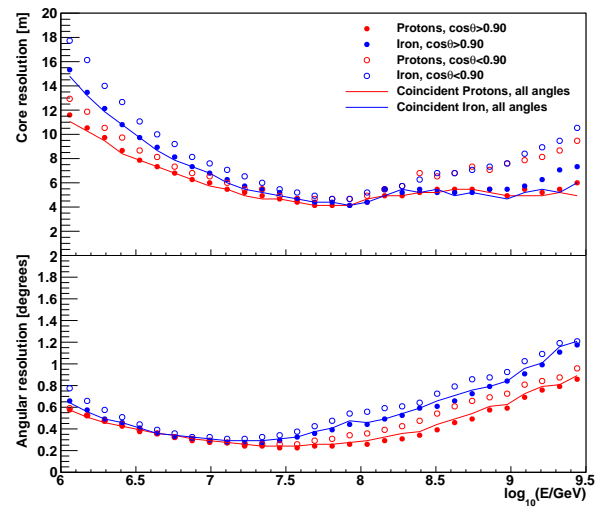

Figure 2: Core position resolution (upper) and angular resolution (lower) of the reconstructed air shower after quality cuts, as a function of primary energy. Defined as containing $68 \%$ of the events. set has a core position resolution of 6-20 meters, and a track direction resolution of 0.31.0 degrees, as a function of energy as shown in Figure 2.

\section{Simulation}

The simulations used in this work are the same as is described in [3]: four primary types (protons, helium, oxygen, and iron) simulated between $\log _{10}(E / \mathrm{GeV})=$ 5.0 and 8.0, as well as thinned simulations stretching up to $\log _{10}(E / \mathrm{GeV})$ of 9.5 . The baseline simulations use CORSIKA-6990, SIBYLL 2.1, and FLUKA. Other high energy hadronic interaction models are used for systematics studies. The IceTop tank simulation is performed by a detailed Geant4 [12] model, and the triggers and readout electronics are also simulated.

Additionally, for the Coincidence analysis, the high-energy muons in these events are propagated to the in-ice detector [13]. The propagation of Cherenkov photons through the South Pole ice from the muons to the DOM's is done using retrieval from tables ("photonics [14]") according to emission angles and distances, followed by simulation of the readout electronics and detector trigger.

In the IceTop simulations used by both analyses, the observation level in CORSIKA was found to be mistakenly set below the height of the snow over the tanks in 13 stations in the northeast corner of the array. As a result, the reconstruction algorithm assumed greater snow attenuation than was actually simulated, and showers landing in the northeast corner were reconstructed with an overestimated $S_{125}$. After reconstructing all events with this issue corrected, new $S_{125}$-primary energy relationships were derived from Monte Carlo and the scale of the energy spectrum is slightly different from [3].

\section{Analysis: IceTop-alone and Coincidence}

In [3], a function relating $\log _{10}\left(S_{125}\right)$ to $\log _{10}(E / G e V)$ was derived using Monte Carlo simu- 
lations divided into many small bins of $\log _{10}\left(S_{125}\right)$, in four ranges of zenith angles and for several different composition assumptions including the "H4a" model ${ }^{1}$ [8], which is repeated here.

The energy resolution and bias of this technique (after the update) is shown in Figure 4(left). Correcting the observation level problem described above results in an improvement in resolution with respect to [3].
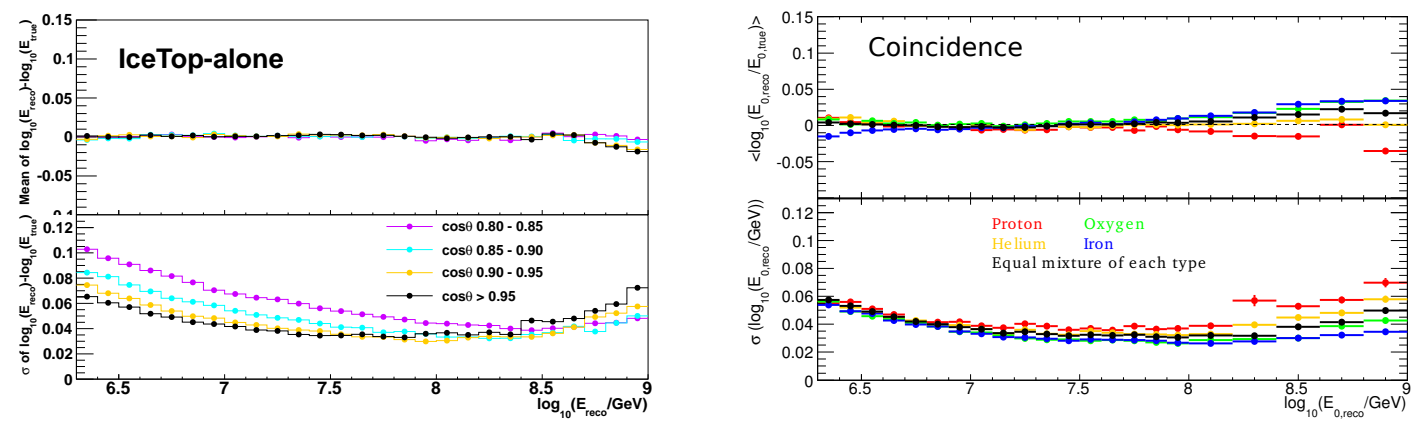

Figure 3: Energy reconstruction bias (top) and resolution (bottom) as a function of the reconstructed energy. For the IceTop-alone analysis (left), an H4a composition mixture is assumed, and four zenith angle bins are shown. For the Coincidence analysis (right), four nuclear types are shown.

In [5, 6], a neural network (NN) was used to estimate primary energy and primary mass using five variables: the shower size in IceTop $S_{125}$, the zenith angle $\cos (\theta)$, the muon energy loss in the ice $d E_{\mu} / d X$ at $X=1500 \mathrm{~m}$, and the number of high-energy stochastics under two selections (standard and strong). In this work, the neural network has been re-optimized and retrained after fixing the observation level problem in the Monte Carlo simulations. The chosen architecture maps the five inputs onto energy and mass using two hidden layers with respectively seven and four neurons (a 5-7-4-2 network). As before, half of the sample is used for the training (25\%) and for testing (another 25\%) the network. The other half (the verification sample) is used for comparing to data in the final stage of the analysis.

The energy dependence for the NN energy bias and NN energy resolution of both proton and iron showers is shown on Figure 4(right). Heavier primaries have a better energy resolution because of their lower intrinsic shower fluctuations. The worsening energy resolution beyond $100 \mathrm{PeV}$ is believed to be caused by the worsening angular resolution which creates an extra smearing in $S_{125}$.

The neural network also reconstructs a natural $\log a r i t h m$ of mass $\langle\log A\rangle$ for each event. Within each bin of reconstructed energy, histograms of this reconstructed $\langle\log A\rangle$ ("template histograms") are constructed for each of the four simulated elemental types, as well as for experimental data. The four types exhibit four distinct shapes in each of the energy bins over the whole energy range. The histogram of NN mass outputs for data events is compared to the set of four template histograms, and the fractions of each which combine to form the data histogram is fitted using a binned likelihood fit that also accounts for Poisson fluctuations in MC [16].

\footnotetext{
${ }^{1}$ Since neither silicon nor magnesium were simulated, oxygen simulation was weighted by the sum of CNO and $\mathrm{MgSi}$ model components.
} 


\section{Systematic Uncertainties}

Both analyses share sources of systematic errors in IceTop. To estimate the effect of the hadronic interaction model, a set was generated using QGSJET-II-03. Because $S_{125}$ is most closely related to primary energy, this IceTop measurement is the dominant source of systematic uncertainty for the spectrum in both analyses. The calibration of the IceTop tanks contains an uncertainty of $3 \%$ on the absolute scale of $S_{125}$. A $0.2 \mathrm{~m}$ uncertainty on the snow attenuation length $\lambda$ affects the $S_{125}$ scale by approximately $3 \%$, which translates to $4-7 \%$ uncertainty in flux.

In the IceTop-alone analysis, a composition model must be assumed, and there is a systematic uncertainty associated with this choice. Investigated in [3], this contribution to the overall systematic errors is isolated in Figure 5.

The dominant systematic on the composition determination is due to uncertainties related to the number of detected photons (the "light yield") in the in-ice detector. The total light yield uncertainty is $+9.6 \% /-12.6 \%$ and has contributions from the DOM efficiency uncertainty (3\%), hole ice uncertainty (3-5\%) and uncertainties related to scattering and absorption properties of the ice $(3-11 \%)$.

\section{Results and Discussion}

Figure 4 shows the result of the IceTop-alone 3 -year analysis, for the 3 years separately on the left, and all combined on the right. This final energy spectrum is compared to the previouslypublished one-year result [3]. A small shift of the spectrum is visible, which is expected after proper treatment of the observation level issue, as discussed in Section 3. Figure 5 shows a similar set of results for the Coincidence analysis, which agrees with the IceTop-alone analysis within the systematic errors.
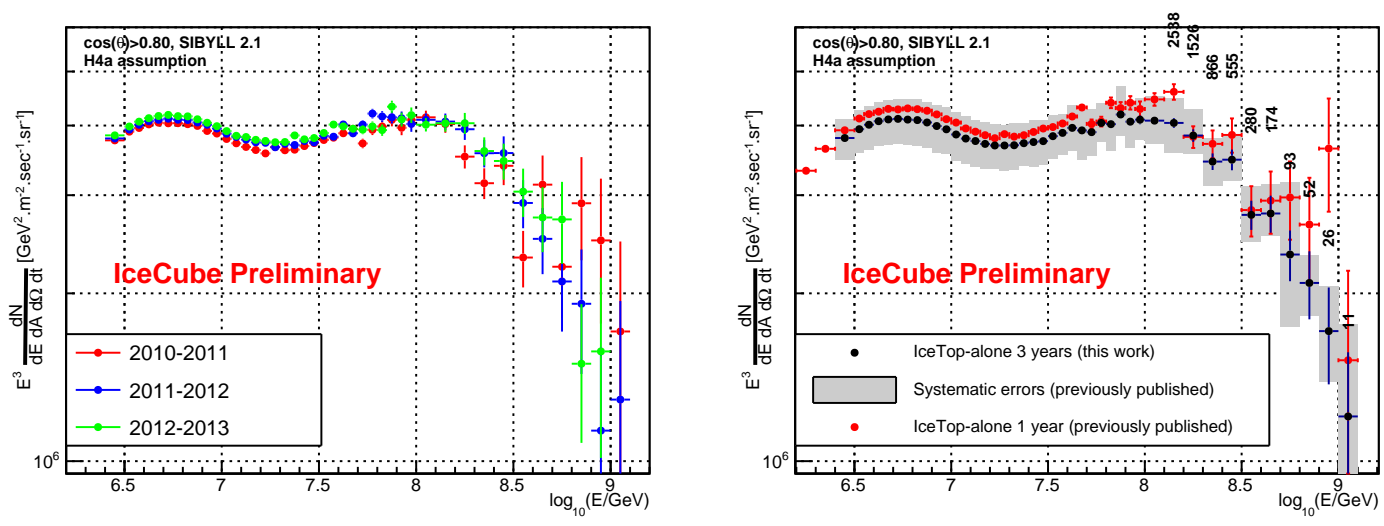

Figure 4: All-particle energy spectrum from the IceTop-alone analysis. Left: the three individual years, and Right: the combined three-year result compared to the previously published one-year result. The grey bands are the systematic errors from the previously published result [3], but applied to the new data points. Increased snow accumulation in the 3-year analysis causes the higher energy threshold with respect to [3].

The features in the energy spectrum seen in [3] are robust: a hardening of the spectrum at around $20 \mathrm{PeV}$, and a softening again past $100 \mathrm{PeV}$. These features are present in both the IceTop- 
alone and Coincidence analyses, and in all three years of data. The energy spectra of the three individual years agree well with each other within their systematic errors. The energy spectra of the two analyses agree within $2 \%$, which is within the estimated systematic error due to the IceTop-alone analysis's assumption of a composition model.

The elemental energy spectra, deduced from multiplying the total energy spectrum with the reconstructed fractions from the template fitting of the $\mathrm{NN}$ mass output, are shown in both panels of Figure 6. We compare the result to alternate results from different systematics shown in grey, in particular: light yield factors (left), and QGSJET-II03 (right), as discussed in Section 5. When broken down by the three individual years of data, both the general features and many of the small-scale fluctuations of these spectra are similar. Despite the large systematic uncertainties, clear differences in behavior between the four elemental groups are visible: protons and helium turning down steeply at

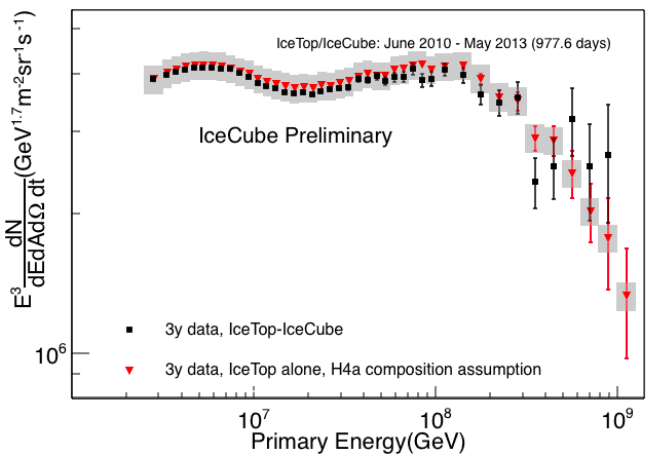

Figure 5: All-particle energy spectrum from the Coincidence analysis, compared to the IceTop-alone result. The grey bound shows the uncertainty due to the unknown composition on the energy spectrum measured by IceTop-alone. lower energies, and oxygen and iron maintaining a harder spectrum up to higher energies.

Figure 7 sums the reconstructed fractions of all four elements weighted with the natural $\operatorname{logarithm}$ of their atomic mass $\langle\log A\rangle$. Systematic uncertainties are represented by alternate $\langle\log A\rangle$ curves on this plot. The average composition increases from the lowest energies up to $\sim 100 \mathrm{PeV}$, where the slope of the trend changes. Approaching $1 \mathrm{EeV}$, there are hints that the average mass is getting lighter again, but in this region the error bars are large and the interpretation ambiguous. Although systematics dominate the absolute scale of the composition measurement, the general trends seen in Figures 6 and 7 are present in the systematics datasets.

\section{References}

[1] A. Achterberg et al., Astropart. Phys. 26 (2006) 155.

[2] R. Abbasi et al., NIM, A700, (2013) 188-220.

[3] M.G. Aartsen et al., Phys. Rev. D, 88, (2013) 042004.

[4] R. Abbasi et al., Astropart. Physics, 42, (2013) 33.

[5] T. Feusels et al., "Cosmic Ray Composition and Energy Spectrum between $2.5 \mathrm{PeV}$ and $1 \mathrm{EeV}$ with IceTop and IceCube", Contributions to the 33rd ICRC (Rio de Janiero), (2013) paper 0861

[6] T. Feusels, Ph.D. thesis, University of Gent (2013)

[7] M. G. Aartsen et al., JINST, 9, (2014) P03009.

[8] T. K. Gaisser, Astropart. Phys, 35, (2012) 801. 

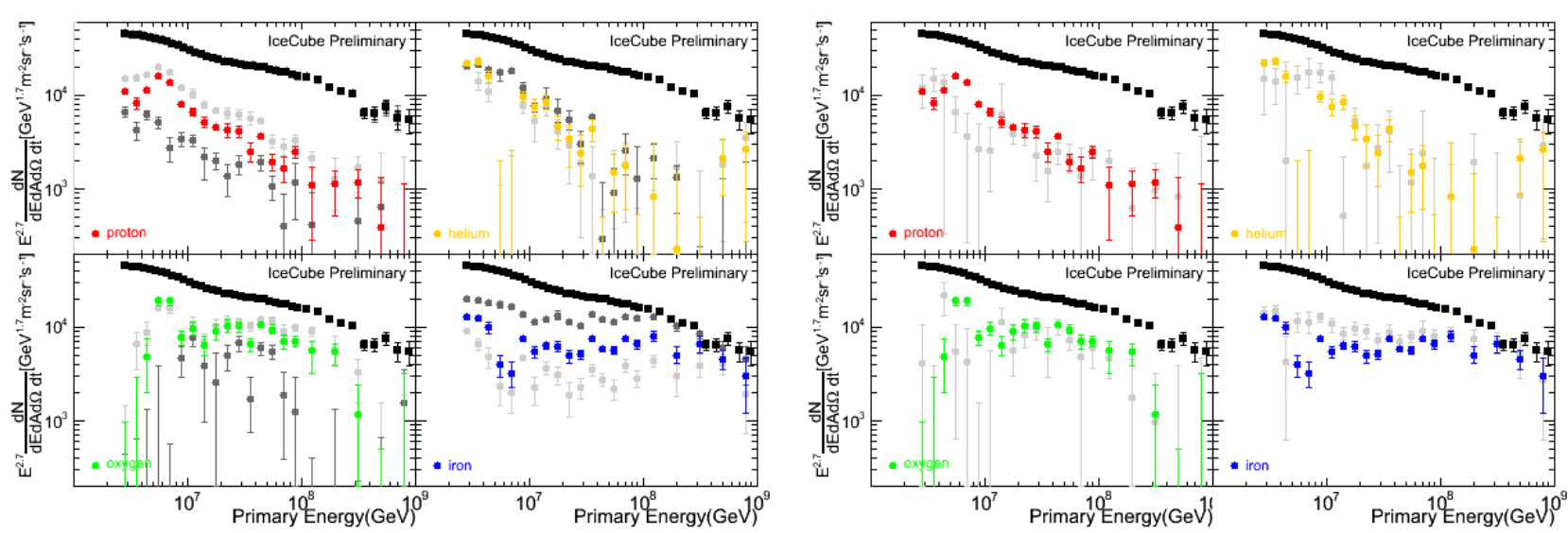

Figure 6: Individual spectra for the four nuclear types (protons, helium, oxygen, and iron), compared with two different sources of systematic uncertainty: the in-ice light yield (dark grey $=-12.5 \%$, light grey $=+9.6 \%$ ) on the left, and QGSJET-II-03 (light grey) as alternate hadronic interaction model on the right. The baseline result (in color) is the same on the left and right.

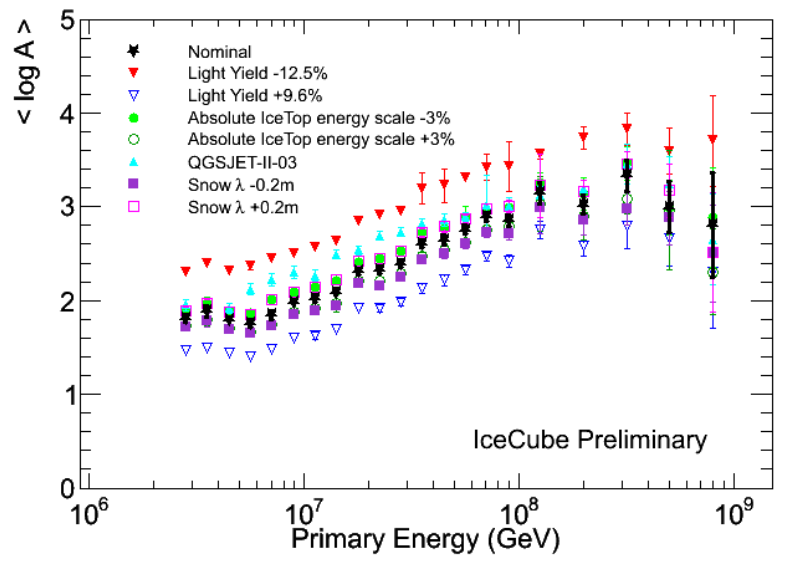

Figure 7: Mean log mass for the three years combined, using baseline simulations (black stars), and systematic uncertainties from alternate simulations represented by other symbols.

[9] D. Heck et al., Report FZKA 6019 (1998).

[10] E. J. Ahn, et al., Phys. Rev. D 80, (2009) 094003.

[11] G. Battistoni et al., AIP Conference proceedings 896, (2007) 31.

[12] S. Agostinelli et al., Nucl. Instrum. Meth. A 506, (2003) 250.

[13] D. Chirkin and W. Rhode "Muon Monte Carlo: A high-precision tool for muon propagation through matter" hep-ph/ 0407075 (2004)

[14] J. Lundberg, P. Miočinović and K. Woschnagg et al., Nucl. Instrum. Meth. A, 581, (2007) 619-631.

[15] S. De Ridder et al., "Seasonal variation of the muon multiplicity in cosmic rays at South Pole", Contributions to the 33rd ICRC (Rio de Janiero), (2013) paper 0763

[16] R. Barlow and C. Beeston, Computer Physics Comm., 77 (1993) 219-228. 